\title{
Nueva colonia reproductiva de foca elefante del sur Mirounga leonina (Linnaeus 1758) (Phocidae) en el sur de Chile
}

\author{
New breeding colony of southern elephant seal \\ Mirounga leonina (Linnaeus 1758) (Phocidae) in southern Chile
}

Juan Capella ${ }^{1,2}$, Frederick Toro ${ }^{3}$, Alejandro Kush ${ }^{4} \&$ Jorge Gibbons $^{5}$

La presencia de foca elefante del sur Mirounga leonina (Linnaus 1758) en el Pacífico Sur Oriental, particularmente en Chile, ha sido escasa durante el siglo XX, con posterioridad a la caza hasta la extinción en 1840 de la colonia reproductiva ubicada en el Archipiélago de Juan Fernández (Philippi, 1892). Más de un siglo después se reportó la existencia de una pequeña colonia reproductiva de la especie, observándose seis a siete animales en el fiordo Parry (54 $40^{\circ} \mathrm{S}$; $6^{\circ} 20^{\circ}$ O), seno Almirantazgo, Tierra del Fuego (Fig. 1) (Markham, 1971), y cuya presencia se limitó a la década de los 70 (Sielfeld, 1978; Torres et al. 1979). Con posterioridad se encontraron otras dos pequeñas colonias reproductivas, ambas en el seno Almirantazgo. Una colonia de 25 a 30 individuos documentada desde 1987 en fiordo Ainsworth

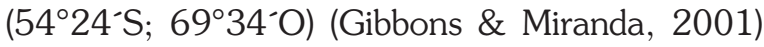
y con evidencia de 2 a 4 crías por temporada entre 2003 y 2014 (Acuña et al. 2005; Cáceres, 2013). Esta pequeña colonia experimentó una disminución del número de ejemplares adultos y crías, con un total de 10 en 2012, hasta reducirse a sólo 3 ejemplares en 2016 (Vila et al. 2015, 2016; Acevedo et al. 2016). En diciembre de 2006 se documentó la presencia de una segunda colonia de 46 ejemplares de foca elefante en bahía Jackson (54 $26^{\prime}$ S) en la cabeza del seno Almirantazgo. Esta colonia creció hasta alcanzar más de 100 ejemplares por temporada entre 2011 y 2016 , con evidencia de nacimientos de dos crías en 2012 y 2013, que posteriormente aumentaron a 15 en 2015, aunque no se observaron crías ni hembras en 2016 (Vila et al. 2016).
En una prospección realizada entre el 27 de febrero y el 3 de marzo de 2014 al estero Poca Esperanza, costa continental de la península Muñoz Gamero, en el golfo Almirante Montt, región de Magallanes, detectamos por primera vez la presencia de 23 ejemplares de foca elefante en caleta Juárez $\left(52^{\circ} 14,0^{\prime} \mathrm{S}-73^{\circ} 07,7^{\prime} \mathrm{O}\right)$, en el extremo sur del brazo oeste del estero (Fig. 1A). La agrupación detectada incluía cuatro crías, una de ellas con pelaje de neonato (negro) (Fig. 2) (Tabla 1). En una visita previa realizada al área el 28 de abril de 2013 pero que no incluyó el desembarco en caleta Juárez, no se registró presencia de la especie.

A partir de ese hallazgo, se estableció un monitoreo de la presencia de foca elefante en caleta Juárez con los objetivos de: i) determinar si la agrupación constituye una colonia reproductiva que regularmente se establece en el lugar, ii) describir su estructura y tamaño y iii) establecer su estacionalidad. Además se realizaron prospecciones

1 Whalesound Ltda, Punta Arenas, Chile.

$\measuredangle$ jjcapella@yahoo.com

2 Fundación Yubarta, Cali, Colombia.

3 Universidad Andrés Bello, Santiago, Chile.

frederick.toro.c@gmail.com

$4 \quad$ Wildlife Conservation Society. akusch@wcs.org

5 Instituto de la Patagonia, Universidad de Magallanes, Punta Arenas, Chile.

jorge.gibbons@umag.cl 


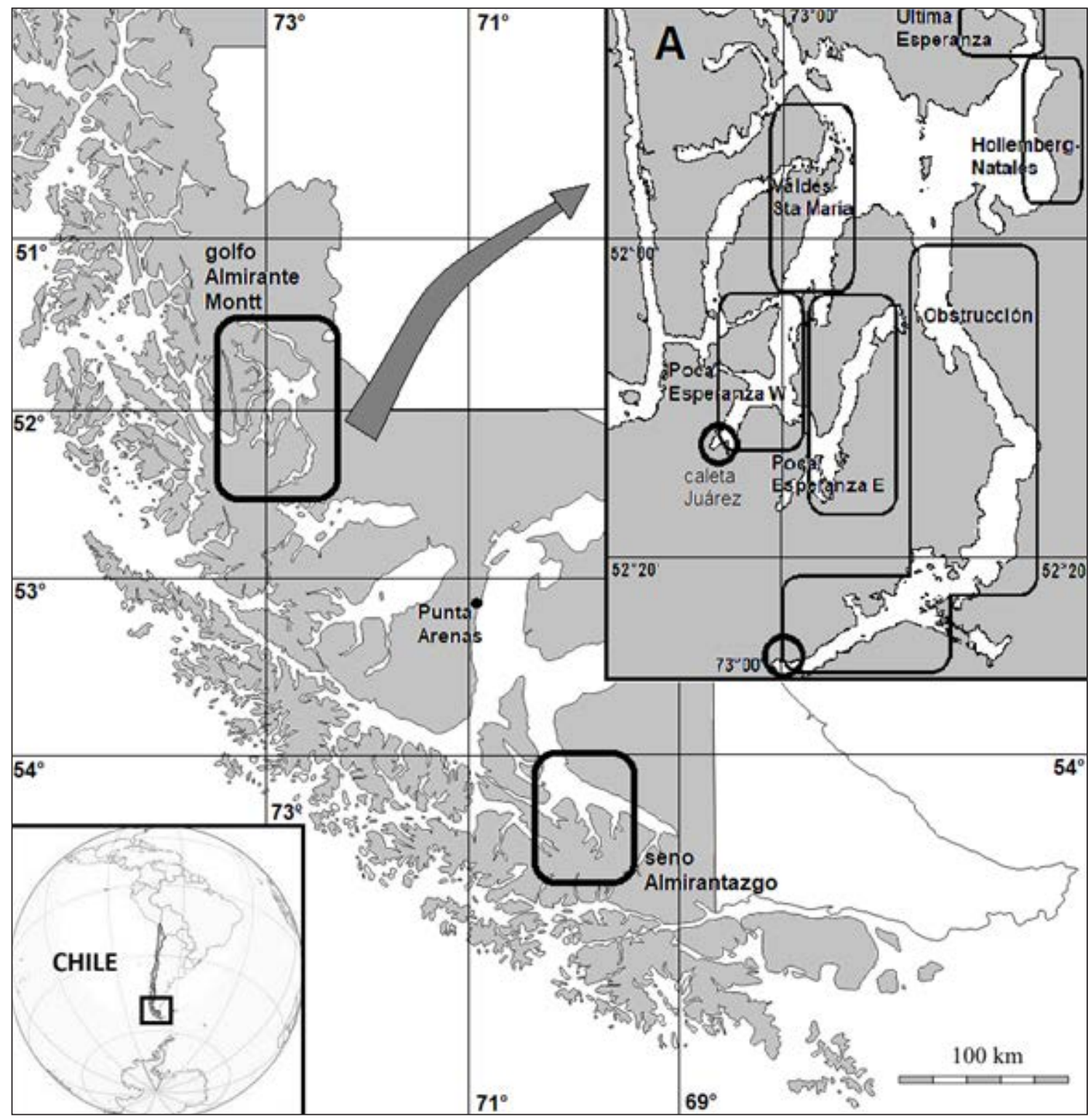

Fig. 1. Áreas de presencia de colonias de foca elefante en el extremo sur de Chile, en el seno Almirantazgo y el golfo Almirante Montt. En el recuadro A se muestran los sectores muestreados en el golfo Almirante Montt y detalle de ubicación de las colonias de foca elefante (círculos) en caleta Juárez (estero Poca Esperanza) y estero Obstrucción.

entre 2014 y 2016 en otros 5 sectores del sistema del golfo Almirante Montt (Fig. 1A) con el objetivo de detectar otras agrupaciones de la especie: Valdés- canal Sta María, brazo este del estero Poca Esperanza, estero Obstrucción, golfo Almirante Montt Este (Hollemberg-Pto. Natales) y estero Última Esperanza.

En caleta Juárez, el área de estudio corresponde a costa conformada por una playa de arena y guijarros que supera los $200 \mathrm{~m}$ de largo, cubierta parcialmente por pastizales y con un humedal costero producido por un río y dos pequeños arroyos que la recorren. Hacia el interior existe abundante vegetación arbórea compuesta principalmente por bosques perennifolios de coigue (Nothofagus betuloides) y canelo (Drymis 
Tabla 1. Número de animales de foca elefante censados en Caleta Juárez entre 2014 y 2016.

\begin{tabular}{|c|c|c|c|c|c|}
\hline Fecha & $\begin{array}{l}\text { Machos } \\
\text { adultos }\end{array}$ & $\begin{array}{c}\text { Hembras } \\
\text { adultas }\end{array}$ & Indeterminados & Crías & Total \\
\hline 02.03 .14 & 5 & 4 & 10 & 4 (1 neonato) & 23 \\
\hline 26.06 .14 & 0 & 0 & 3 & 0 & 3 \\
\hline 09.07 .14 & 0 & 0 & 3 & 0 & 3 \\
\hline 29.07 .14 & 0 & 0 & 1 & 0 & 1 \\
\hline 06.09 .14 & 0 & 0 & 0 & 0 & 0 \\
\hline 22.09 .14 & 0 & 0 & 4 & 0 & 4 \\
\hline 10.10 .14 & 0 & 0 & 5 & 0 & 5 \\
\hline 21.10 .14 & 0 & 0 & 3 & 0 & 3 \\
\hline 20.11 .14 & 4 & 3 & 13 & 2 neonatos & 22 \\
\hline 19.02 .15 & 8 & 2 & 0 & 2 & 12 \\
\hline 20.02 .15 & 1 & 2 & 7 & 2 & 12 \\
\hline 30.03 .15 & 4 & 0 & 27 & 2 & 33 \\
\hline 24.09 .15 & 6 & 2 & 0 & 2 neonatos (1 muerto) & 10 \\
\hline 21.11 .15 & 11 & 4 & 3 & 3 neonatos & 21 \\
\hline 30.11 .15 & - & - & - & 3 neonatos (1 muerto) & 44 \\
\hline 16.12 .16 & 8 & 0 & 34 & 4 (1 neonato) & 46 \\
\hline
\end{tabular}

winteri) que se densifican tierra adentro.

Se realizaron 16 jornadas de muestreo para monitorear la colonia de caleta Juárez, entre marzo de 2014 y diciembre de 2016 (Tabla 1). Las observaciones se llevaron a cabo desde tierra, a simple vista y con la ayuda de binoculares $8 \times 35$. Durante el período de observación se realizaron censos de foca elefante, identificando cuando fue posible a machos adultos, hembras adultas y crías, y categorizando como indeterminados cuando no fue posible la discriminación de los individuos. Los conteos incluyeron a ejemplares apostados en la playa, en el agua y dentro del área interior de bosque denso. Los animales ubicados en la playa o en zonas despejadas permitían nuestra aproximación a corta distancia para su identificación sin mostrar perturbación aparente pues permanecían echados, no cambiaban de posición o continuaban durmiendo. En cambio los que se ubicaban al interior del tupido bosque parecían más sensibles al acercamiento debido al ruido y movimiento de ramas al caminar, lo que producía la alerta y movimiento de los animales. El mantenernos a distancia para no perturbar a la colonia limitó nuestra visibilidad en el sector interior, lo que impidió la determinación de clases de animales y en algunos casos el conteo de ejemplares.

Para las prospecciones realizadas en otros sectores del golfo Almirante Montt (ver Fig. 1A) entre 2014 y 2016 se utilizó una embarcación menor navegando cercana a la costa (a no más de $200 \mathrm{~m}$ de la orilla) y a baja velocidad (10 km/h). El muestreo se llevó a cabo con dos observadores apoyados con el uso de binoculares $8 \times 35$. En caso de avistar a la foca elefante, de efectuó el desembarco para el conteo de los animales, los que se identificaron en las mismas categorías utilizadas en caleta Juárez.

Los resultados del monitoreo mostraron que:

a) La agrupación de foca elefante de caleta Juárez es reproductiva, siendo la colonia más septentrional del Pacífico Sur Oriental, distante $325 \mathrm{~km}$ al norte de las conocidas en el seno Almirantazgo. Se constató la presencia de crías en tres temporadas reproductivas consecutivas con un máximo de 4 crías/ temporada (Tabla 1). Varios de los registros de crías fueron neonatos: uno en marzo de 2014, dos en noviembre de 2014, dos en 
septiembre (incluyendo una muerta) y tres en noviembre de 2015 (una muerta, hecho ocurrido entre el 21 y el 30 de noviembre) y una en diciembre de 2016 (Tabla 1).

b) La estructura de la población no ha podido ser descrita en detalle debido a que un número importante de animales fue clasificado como indeterminado (61.5\%) pues se ubicaban dentro del bosque y se dificultaba la observación directa y la aproximación sin causar perturbación. Los resultados muestran un número creciente de animales cada año en la estación de primavera y verano, con la excepción de un censo en primavera del 2015 en que se obtuvo el mínimo de las cuatro temporadas. El bajo número de ejemplares contabilizado el 21 de noviembre pudo deberse a que el monitoreo de la colonia se realizó 4 días después del aterrizaje de un helicóptero en la playa de caleta Juárez. Esta interpretación se respalda en la siguiente observación realizada el 30 de noviembre por personal de la empresa Salmones Magallanes que se encontraba limpiando esta playa y detectó la presencia de 44 ejemplares y un neonato muerto (A. Díaz com pers). Este conteo muestra una gran similitud con el realizado con posterioridad en diciembre de 2016 (46). El número total de animales se incrementó, duplicándose en la última temporada en comparación al primer conteo de 2014, lo que indica el aumento de la población. Sin embargo, el intervalo de tiempo monitoreado (2014-2016) y el alto número de ejemplares indeterminados no permiten hacer inferencias sobre la dinámica poblacional de la colonia.

c) La especie fue detectada en caleta Juárez durante todas las estaciones del año y en todas las visitas realizadas, con la excepción de la del 28 de abril de 2013, fecha en que no se realizó un desembarco, y la del 6 de septiembre de 2014. La mayor abundancia se observó entre noviembre y marzo (promedio 27,1; rango 12-46 animales), meses que corresponden a la primavera y el verano, mientras que los menores conteos de ejemplares ocurrieron

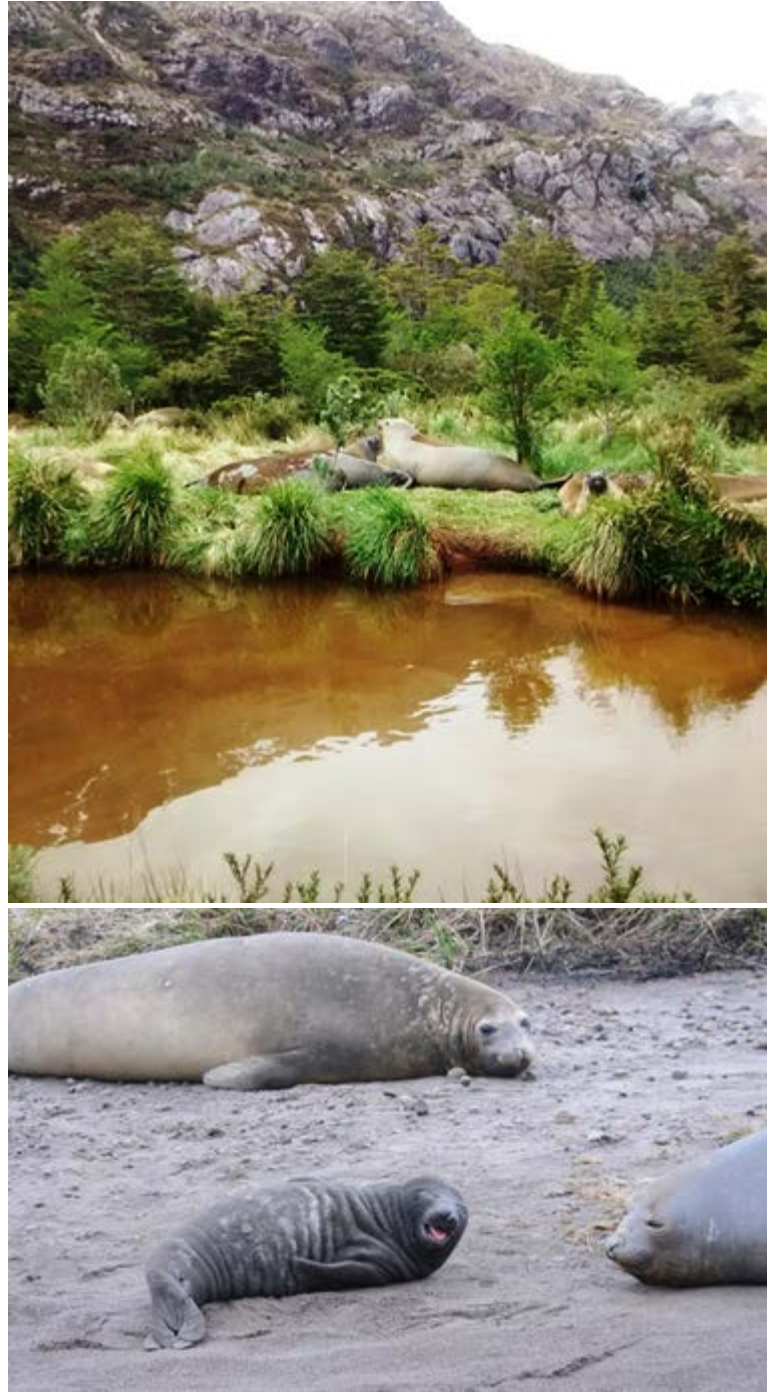

Fig. 2. Vista de la colonia de foca elefante de caleta Juárez (foto superior) y presencia de un neonato y hembra (foto inferior).

entre mediados de otoño y comienzos de la primavera, con un promedio de 3,6 y un rango de 0 - 10 animales. La estacionalidad observada es consistente con el ciclo de vida descrito para la foca elefante, conformado por dos etapas terrestres, la de reproducción y la de muda, y períodos de alimentación en el mar (Lewis, 1996). La detección de neonatos entre septiembre y diciembre $y$ de crías pequeñas hasta fines de febrero es consistente con lo observado por Campagna et al. (1993). Estos autores indican que la 
temporada reproductiva para la colonia de Península Valdés, Argentina, comienza durante la tercera semana de agosto, cuando los primeros machos y hembras llegan a la costa, y se extiende hasta noviembre. En caleta Juárez se han observado animales mudando en verano, desde diciembre hasta comienzos de marzo, lo que coincide con la observación de ejemplares en proceso de muda durante febrero en bahía Jackson (Vila et al. 2016).

d) En prospecciones costeras realizadas entre los años 2014 y 2016, en otros 5 sectores del sistema del golfo Almirante Montt (Fig. 1A), fue posible detectar la presencia de otra agrupación de foca elefante en la cabeza del estero Obstrucción (52²9' S - 7259'O) (Fig. 1A). Esta agrupación estaba constituida por un total de 8 ejemplares, de los cuales se pudo determinar un macho adulto muerto y un juvenil. La presencia de foca elefante para el estero Obstrucción también fue registrada en octubre de 2016 (John Gibbons, com pers) y Acevedo et al. (2016) mencionan el registro de una hembra con su cría en octubre de 2010 pero en un sector intermedio del estero, cercano a una zona poblada. Todo lo anterior sugiere que también se trataría de una colonia reproductiva.

Nuestros resultados indican que la agrupación de foca elefante establecida en caleta Juárez corresponde a una nueva colonia reproductiva que usó repetidamente el área, que en el período de tiempo monitoreado duplicó el número de ejemplares establecidos allí y que constituye la colonia más septentrional existente en Chile. Las características geomorfológicas y ambientales del sitio donde se encuentra esta colonia muestran una pendiente casi nula del sustrato, una playa de material arenoso fino flanqueada por un humedal costero de agua dulce, y una vegetación boscosa que se densifica hacia el interior.

La colonia de foca elefante de caleta Juárez presentaría una baja perturbación antrópica (los animales en las zonas abiertas no mostraban inquietud ni reaccionaban a nuestra aproximación), con la excepción de episodios como el del descenso de un helicóptero ya mencionado. Tres aspectos respaldan la afirmación anterior: i) presencia regular de la especie y el incremento del número de ejemplares en la colonia en el período muestreado, ii) la constancia de evidencias de reproducción a lo largo de cuatro temporadas reproductivas, iii) la baja perturbación aparente en la conducta de los individuos ante presencia humana cautelosa en las playas.

Considerando la ubicación de esta colonia de Poca Esperanza, su cercanía a Puerto Natales centro del turismo regional, y el carácter carismático de la especie como atractivo turístico, se configura un escenario probable de alta demanda de visitación futura y probable perturbación, lo que conlleva un evidente riesgo para su conservación si no se aplican buenas prácticas de turismo sustentable y controles adecuados (Cáceres, 2013). Se ha mencionado que el turismo no regulado podría haber sido una causa probable de la desaparición de la colonia de fiordo Ainsworth en seno Almirantazgo (Cáceres, 2013). Vila et al. (2016) demuestran mediante seguimiento satelital de ejemplares de foca elefante que existe conectividad entre las colonias de seno Almirantazgo y de caleta Juárez. Dicha conectividad entre las colonias establece, en principio, que habría una unidad poblacional en la región, lo que implica entonces que el riesgo identificado sería compartido entre las diferentes colonias.

La relevancia y urgencia de disponer de monitoreos de largo plazo, estudios de identidad poblacional e implementar estrategias de conservación para la colonia de foca elefante de caleta Juárez, así como para las otras agrupaciones conocidas de la especie en la región austral de Chile es pertinente y necesaria, entre otras, para dar claridad respecto a dos interesantes hipótesis recientemente sugeridas por Acevedo et al. (2016) respecto al origen y expansión de la distribución de la foca elefante del Pacífico Sureste: 1) que la recolonización de la foca elefante en la costa de Chile luego de 130 años desaparecida (1840-1971) puede deberse a una inmigración en sentido sur a norte que habría comenzado al sur de los $51^{\circ} \mathrm{S}$ y 2) que análisis preliminares de $\mathrm{ADN}$ mitocondrial de la foca elefante de la región muestran una alta diversidad de haplotipos en la población, de los cuales una fracción menor se relaciona con las colonias existentes en Las Malvinas pero la 
mayoría son de origen desconocido, por lo que se sugiere el posible resurgimiento a partir de algunos remanentes remotos de la población histórica del Pacifico Sur Oriental que habitaba la costa chilena continental, y se reproducía en el Archipiélago de Juan Fernández, para la cual no existe una caracterización genética.

\section{AGRADECIMIENTOS}

El monitoreo del área y el estudio de la foca elefante contó con el apoyo logístico y financiero de Salmones Magallanes, S.A. Un reconocimiento al personal de los centros de la empresa en el sector por su hospitalidad. Se agradece también la información aportada por el Sr. John Gibbons, la colaboración en terreno de Emiliano Méndez y Jaime Cárcamo, las facilidades y equipos del laboratorio de Hidrobiología del Instituto de la Patagonia y las sugerencias realizadas por los revisores de este trabajo.

\section{LITERATURA CITADA}

Acevedo J., Aguayo-Lobo, A., Brito, J., Torres, D., Caceres, B., Vila, A., Cardeña, M., \& Acuña, P. (2016). (Mirounga leonina): a review of the current distribution of southern elephant seal in the eastern South Pacific. New Zealand Journal of Marine and Freshwater Research, 50, 240-258.

Acuña P., Aguayo-Lobo, A., Acevedo, J., Parra, G., \& Pastene, P. (2005). Origen de Mirounga leonina en Seno Almirantazgo, Chile. Book of Abstracts, in Internacional Symposium, New Approaches to the Quaternary Sciences in Fuego-Patagonia.

Cáceres, B. (2013). Comportamiento reproductivo de la foca elefante Mirounga leonina (Linnaeus 1758) en bahía Aisnworth, Tierra del Fuego y los potenciales efectos del turismo. Tesis de Grado Biología Marina, Universidad Austral, Chile.

Campagna, C., Lewis, M., \& Balde, R. (1993). Breeding biology of southern elephant seals in Patagonia. Marine Mammal Science, 1,
$34-47$

Gibbons, J., \& Miranda, C. (2001). Southern elephant seal (Mirounga leonina) (PHOCIDAE) at Almirantazgo Sound, Tierra del Fuego. Anales del Instituto de la Patagonia, Serie Ciencias Naturales, 29, 157-159.

Lewis, M. (1996). El elefante marino del sur. Biología de la especie, descripción general de la agrupación de la Península Valdés y protocolos de trabajo. Informes Técnicos del Plan de Manejo Integrado de la Zona Costera Patagónica (Puerto Madryn, Argentina).

Markham, B. (1971). Observaciones sobre el elefante marino del sur y el leopardo marino de la península Brecknock (Parque Nacional "Alberto M. de Agostini"), Tierra del Fuego, Anales del Instituto de la Patagonia, Punta Arenas, Chile, 9, 153-156.

Philippi, R. A. (1892). Las focas chilenas del Museo Nacional figuradas i descritas. Anales del Museo Nacional de Chile, Primera Sección I Zoología.

Sielfeld, W. (1978). Algunas consideraciones sobre fócidos (Pinnipedia) asociados a las costas de Chile. Anales del Instituto de la Patagonia, 9, 153-156.

Torres, D, Yánez, J., \& Cattan, P. (1979). Mamíferos marinos de Chile: Antecedentes y situación actual. Biología Pesquera, 11, 49-81.

Vila, AR., Uhart, M., Droguett, D., Silva-Quintas, C., Muza, R., \& Farías, F. (2015). Estudio de la ecología y estado sanitario de foca elefante del sur (Mirounga leonina) en Seno Almirantazgo, Tierra del Fuego. Informe preparado para la Subsecretaria de Pesca, RS No. 2911 de 2012, Punta Arenas.

Vila, A. R., Kusch, A., Munzenmayer, R., \& Terán, D. (2016). Estudio de la ecología $y$ estado sanitario de foca elefante del sur (Mirounga leonina) en Seno Almirantazgo, Tierra del Fuego. Informe preparado para la Subsecretaria de Pesca, RS No 380 de 2016, Punta Arenas. 